\title{
Sudden postoperative death caused by unheralded Mallory Weiss tears
}

\author{
G M E Humphrey, E W Benbow, W F Tait
}

\begin{abstract}
Mallory Weiss tears are a common cause of upper gastrointestinal bleeding, typically reported as following repeated vomiting after an alcoholic binge. This association may have been overemphasised, and these lesions could be caused by a wide range of spontaneous and iatrogenic events. A case of sudden postoperative death caused by massive haematemesis, unheralded by any evidence of vomiting or retching, as a result of Mallory Weiss tears is reported.
\end{abstract}

Haematemesis caused by laceration of the mucosa of the oesophagogastric junction was described by Quincke in $1879,{ }^{1}$ and its association with vomiting and alcohol consumption was reported by Mallory and Weiss in $1929 .^{2}$ Since then, the association has become known as the Mallory Weiss syndrome. Initially thought to be rare, the advent of endoscopy has facilitated recognition of the mucosal lesions, and it is now recognised that they cause $5-15 \%$ of all episodes of upper gastrointestinal bleeding. ${ }^{3-6}$ It has also been realised that there are various other predisposing conditions.

We report a case of fatal gastrointestinal haemorrhage caused by Mallory Weiss tears, occurring without warning during otherwise uneventful postoperative recovery. We can find no published report of a similar case.

\section{Case report}

A 51 year old woman was admitted with an acutely ischaemic leg following occlusion of a right axillofemoral graft earlier that day. She had remained well since her last vascular surgery in 1987. There were no signs or symptoms of upper gastrointestinal disease. She was taking Augmentin (Beecham) for a recent urinary tract infection. Previous investigation had shown no risk factors for arterial disease apart from smoking. Her haemoglobin concentration was $11.2 \mathrm{~g} / \mathrm{l}$; urea and electrolytes were normal.

At surgery the right axillofemoral graft was thrombosed, and a new right axillofemoral graft was inserted successfully. There was no manipulation of the upper gastrointestinal tract before, during, or after surgery. Postoperatively, she was given dipyridamole but was not anticoagulated.

On the second postoperative day she complained of nausea and was given prochlorperazine $12.5 \mathrm{mg}$ intramuscularly. Later that evening she sustained a massive haematemesis and died within an hour despite

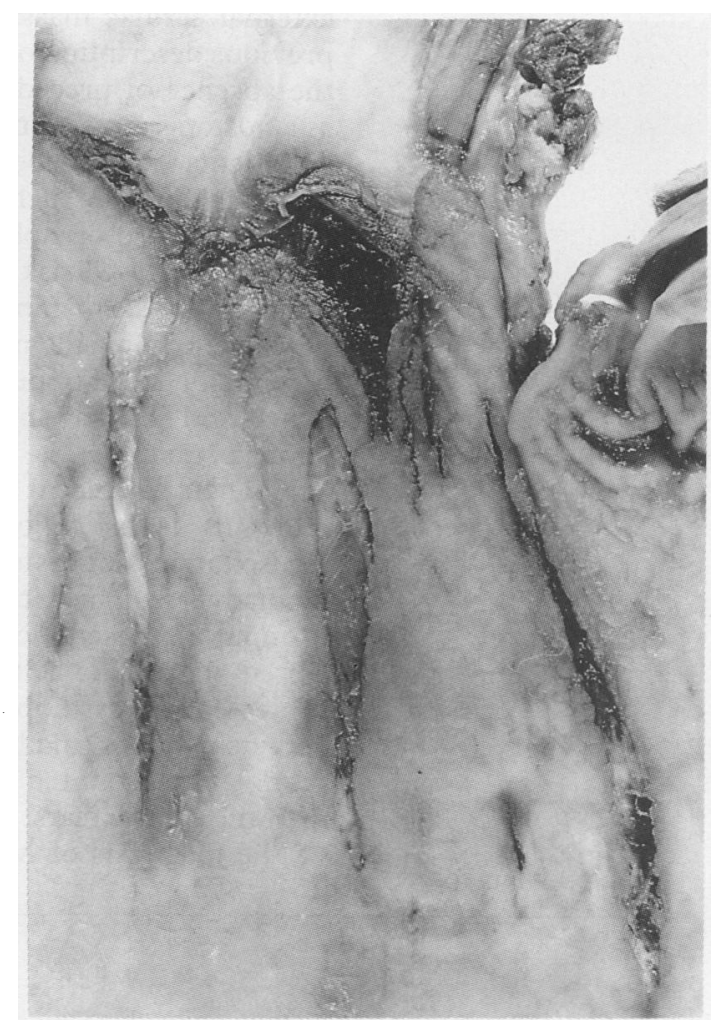

Cardio-oesophageal junction with several longitudinal tears in the mucosa.

resuscitation, and before endoscopy or surgery could take place. No vomiting or retching had been observed prior to the haematemesis.

\section{Pathology}

At necropsy there was about $1500 \mathrm{ml}$ of fresh blood and blood clot in the lumen of the stomach, with more fresh blood filling the lumen of the entire oesophagus and much of the small intestine. There were several ragged tears in the mucosa at and below the cardio-oesophageal junction (figure).

All vascular anastomoses were intact; in particular, there was no evidence of an aortoenteric fistula.

\section{Discussion}

Although the diagnosis of Mallory Weiss syndrome was originally restricted to cases in which haematemesis followed vomiting, usually in association with an alcoholic binge, ${ }^{2}$ the diagnostic criteria have since been widened to include haematemesis as a result of mucosal tearing, regardless of the precipitating event. ${ }^{6}$ In particular, the roles of alcohol and preceding vomiting have been overemphasised. 
Mallory Weiss tears have been reported in association with many conditions, including vomiting caused by raised intracranial pressure, pregnancy, cytotoxic drugs, and ipecac. Other recognised precipitating events include straining at passing stools, hiccoughing, protracted coughing, snoring and blunt abdominal trauma. Medical and surgical procedures have also been implicated, including gastroscopy, transtracheal aspiration, and external cardiac massage, but we can find no previous description of Mallory Weiss tears, in the absence of preceding vomiting, occurring as a postoperative complication.

Patients with coexistent upper gastrointestinal disease, such as hiatus hernia, peptic ulceration, and oesophagitis or gastritis, seem to be at increased risk, and an association with non-steroidal inflammatory agents has been described. Dipyridamole is associated with a high incidence of upper gastrointestinal disturbance ${ }^{7}$ and may have been an aggravating factor in our patient.

Hulewicz's recent description of gastric mucosal tears following cardiopulmonary resuscitation ${ }^{8}$ suggested that they were caused by inadvertent gastric inflation during mouthto-mouth resuscitation of patients who collapsed in public. Although our patient was subjected to cardiopulmonary resuscitation, her haematemesis had occurred before this and cannot be accounted for by any lesion other than the gastric tears.

The diagnosis of Mallory Weiss lesions on clinical grounds alone is difficult because of the wide range of predisposing factors and variations in presentation, correct pre-endoscopy diagnosis being made in only $36 \%$ of cases. ${ }^{9}$ These lesions are the third commonest cause of upper gastrointestinal haemorrhage after duodenal ulceration and gastric ulceration, ${ }^{10}$ and the fact that they can cause fatal haematemesis without any warning should be emphasised.

We thank $\mathrm{Mr} \mathrm{R}$ W $\mathrm{G}$ Johnson, consultant surgeon, for permission to report the clinical details, Mr L M Gorodkin, HM Coroner for Greater Manchester (Central), for permission to report the pathological aspects, and Mrs J Crossley for taking the photograph.

1 Quincke H. Ulcus oesophagi ex digestion. Dtsh Arch Klin Med 1879;24:72.

2 Mallory GK, Weiss S. Hemorrhage from laceration of the cardiac orifice due to vomiting. Am J Med Sci 1929; 178:506.

3 Watts HD, Admirand WH. Mallory Weiss syndrome. A reappraisal. $J A M A$ 1974;230:1674-5.

4 Graham DY, Schwartz JT. The spectrum of Mallory Weiss. Medicine 1977;57:307-18.

5 Graham DY, Davis RE. Acute upper GI hemorrhage. New observations of an old problem. Am J Dig Dis 1978;23:876.

6 Knauer CM. Mallory Weiss syndrome: characteristics of 75 Mallory Weiss lacerations in 528 patients with upper gastrointestinal haemorrhage. Gastroenterology 1976, 71:5-8.

7 Browse NL, Hall JN. Effect of dipyrimidole on the incidence of clinically detectable deep vein thrombosis. Lancet 1969;ii:718-20.

8 Hulewicz B. Gastric trauma following cardiopulmonary resuscitation. Med Sci Law 1990;30:149-53.

9 Hastings PR, Peters KW, Cohn I. Mallory Weiss syndrome: review of 64 cases. Am J Surg 1981;142:560-2.

10 Sugawa C, Benishek D, Walt AJ. Mallory Weiss syndrome. A study of 224 patients. Am J Surg 1985;145:30-3.

\footnotetext{
Department of Haematology, University College and Middlesex School of Medicine, London WC1E 6AU

C Christopoulos C Mattock

Correspondence to: Dr C Christopoulos Accepted for publication 20 February 1991
}

\begin{abstract}
Blood smears from a patient with severe generalised arteriopathy and an occluded synthetic femoropopliteal graft showed the phenomenon of EDTA dependent adherence of platelets to neutrophils (platelet satellitism). Immunoenzymatic staining with a monoclonal antibody to thrombospondin showed that adherence to neutrophils exclusively involved platelets that stained strongly positive for thrombospondin, while negative or weakly positive platelets showed no tendency to adhere. There was no increase in platelet surface immunoglobulins. This suggests a possible role for thrombospondin or some other cytoadhesive platelet $a$ granule protein in mediating the adherence of platelets to neutrophils in cases of satellitism.
\end{abstract}

Adherence of platelets to neutrophils in a rosette formation, known as platelet satellitism, is a rare in vitro phenomenon typically seen in EDTA anticoagulated blood. It is not consistently related to any clinical entity but can be the cause of spurious thrombocytopenia. The exact mechanism of this phenomenon is unknown but its transferability, at least in some cases, to normal blood by patients' serum ${ }^{12}$ and a report of increased surface IgG in the adhering platelets ${ }^{3}$ suggest that immunoglobulins have a central role. Our findings in the following case of satellitism point toward an alternative link that might be mediating the adherence of platelets to neutrophils in this setting.

\section{Case report}

A 69 year old woman was admitted for replacement of an occluded synthetic femoropopliteal arterial graft. Apart from severe peripheral arteriopathy she also had ischaemic heart disease, having had a triple by-pass graft two years previously. Aspirin had been discontinued three days before admission. A routine preoperative blood count (EDTA anticoagulant) showed a normal platelet count but the white cell differential 